\title{
Características demográficas e psicossociais associadas à depressão pós-parto em uma amostra de Belo Horizonte
}

\author{
Demographic and psychosocial characteristics associated with postpartum depression in \\ a sample from Belo Horizonte
}

\section{Patricia Gomes Figueira ${ }^{1}$, Leandro Malloy Diniz ${ }^{2}$, Humberto Correa da Silva Filho ${ }^{2}$}

${ }^{1}$ Mestre. Médica psiquiatra. Programa de Pós-Graduação em Neurociências, Universidade Federal de Minas Gerais (UFMG), Belo Horizonte, MG. ${ }^{2}$ Doutor. Professor, Departamento de Saúde Mental, UFMG.

Suporte financeiro: Fundação de Amparo à Pesquisa do Estado de Minas Gerais (FAPEMIG) e Conselho Nacional de Desenvolvimento Científico e Tecnológico (CNPq).

\section{Resumo}

Introdução: A depressão pós-parto (DPP) tem sido alvo de inúmeras investigações científicas devido à sua alta prevalência e gravidade e também por suas repercussões negativas na puérpera e na criança.

Objetivo: Comparar mulheres com e sem DPP em um grupo de puérperas selecionadas aleatoriamente a partir dos partos ocorridos em uma maternidade de Belo Horizonte (MG).

Métodos: Selecionamos 245 mulheres que tiveram parto em uma maternidade de Belo Horizonte. Aplicamos uma entrevista semiestruturada para a obtenção de dados psicossociais e demográficos e uma entrevista estruturada (Mini Neuropsychiatric Interview, MINI-Plus) para o diagnóstico de depressão maior segundo critérios do Diagnostic and Statistical Manual of Mental Disorders, 4th edition (DSM-IV).

Resultados: $26,9 \%$ das mulheres tinham diagnóstico de DPP. Não encontramos diferenças entre as características sociodemográficas das mulheres $\operatorname{com}(\mathrm{n}=66,26,9 \%)$ e sem diagnóstico de DPP $(\mathrm{n}=179,73,1 \%)$. Entretanto, diversas variáveis clínicas e psicossociais se mostraram significativamente diferentes entre os dois grupos, tais como história de depressão, vivência de estresse ou presença de sintomas depressivos ou ansiosos durante a gravidez, ocorrência de complicações maternas ou na criança no pós-parto e insuficiência de suporte nos cuidados pós-natais.

Conclusão: A identificação de fatores associados à DPP é importante para a compreensão de sua etiopatogenia e para o estabelecimento de estratégias de prevenção e tratamento precoce dessa grave doença.

Descritores: Depressão pós-parto, prevalência, fatores de risco.

\begin{abstract}
Introduction: Postpartum depression has been the object of extensive research because of both its high prevalence and its repercussions on the mother and the infant.

Objective: To compare women with and without depression in a sample of postpartum women randomly selected at a maternity ward in the city of Belo Horizonte, Brazil.

Methods: A total of 245 women who gave birth at a private maternity hospital in Belo Horizonte were selected. A semi-structured interview was used for the collection of psychosocial and demographic data. Diagnosis of major depression was established using and a structured interview (Mini Neuropsychiatric Interview, MINI-Plus), based on criteria set forth in the Diagnostic and Statistical Manual of Mental Disorders, 4th edition (DSM-IV).

Results: Of the total sample, $26.9 \%$ had a diagnosis of postpartum depression. No differences were observed in the social and demographic characteristics of depressed women $(n=66,26.9 \%)$ and of those without depression $(n=179,73.1 \%)$. Nevertheless, several clinical and psychosocial variables showed significant differences between the groups and were therefore associated with postpartum depression, namely previous history of depression, presence of stress or depressive/anxiety symptoms during pregnancy, postpartum complications affecting the mother or the infant, and lack of support and care in the postpartum period.

Conclusion: The identification of factors associated with postpartum depression is important for an improved understanding of the pathophysiology of this disease and for the establishment of strategies aimed at prevention and early diagnosis.
\end{abstract}

Keywords: Postpartum depression, prevalence, risk factors.

\section{Correspondência:}

Patricia Gomes Figueira, Rua Gustavo Pena, 44/504, Horto, CEP 31015-060, Belo Horizonte, MG, Brasil. Tel.: (31) 3482.8003. E-mail: patriciagfigueira@gmail.com Não foram declarados conflitos de interesse associados à publicação deste artigo.

Copyright (C) Revista de Psiquiatria do Rio Grande do Sul - APRS

Recebido 06/07/2010. Aceito em 13/07/2010. 


\section{Introdução}

A depressão pós-parto (DPP) é um transtorno psiquiátrico com várias repercussões negativas sobre a paciente, a criança e as relações familiares ${ }^{1}$. A DPP pode durar vários anos e está relacionada a aumento de risco de outros quadros depressivos recorrentes ${ }^{2}$. O impacto sobre a criança também é significativo, pois a doença limita a habilidade da mãe de responder à demanda envolvida nos cuidados e sua capacidade de engajamento positivo ${ }^{3}$ e contato emocional com o bebêt $\hat{e}^{4}$ Filhos de mães deprimidas podem apresentar dificuldades no desenvolvimento emocional e comportamental, bem como atraso no desenvolvimento cognitivo e no ganho ponderal ${ }^{1,4-6}$. Estudos de observação 'mãe-bebê' mostram que recém-nascidos de mães com DPP exibem menos expressões faciais positivas e mais expressões faciais negativas, mais comportamento de protesto, mais irritabilidade, menos vocalizações e menor atividade física ${ }^{1}$. Tais prejuízos persistem, com vários estudos evidenciando pior desenvolvimento cognitivo e mais dificuldades emocionais em crianças de 12 a 21 meses cujas mães apresentaram $\mathrm{DPP}^{7,8}$.

Os dados relativos à prevalência da DPP são discrepantes. No Brasil, foi encontrada uma prevalência de $12 \%$ no Rio de Janeiro ${ }^{9}, 13,4 \%$ em Brasília $^{10}$, 20,7\% em Porto Alegre ${ }^{11}$, 26.9\% em Belo Horizonte ${ }^{12}$ e 7,2\% em Recife ${ }^{13}$. Uma revisão da literatura incluindo 143 estudos encontrou prevalências variando de 0 a $60 \%$;4; já uma metanálise que incluiu 59 estudos realizados em todo o mundo observou uma prevalência média de $13 \%{ }^{15}$.

Do ponto de vista etiológico, coexistem dois modelos explicativos, provavelmente complementares. Um modelo biológico, que sustenta a existência de uma vulnerabilidade hormonal ${ }^{16} \mathrm{e} / \mathrm{ou}$ genética $^{17}$, e um modelo psicossocial, que postula que as transformações na vida da mulher após o parto contribuiriam para o desencadeamento da depressão ${ }^{18}$. Alguns estudos, incluindo um realizado no Brasil ${ }^{13}$, procuraram identificar características ou fatores de risco associados à DPP. Dentre esses fatores de risco, os principais encontrados foram: ser solteira, história de síndrome pré-menstrual, ocorrência de disforia no pós-parto, sintomas depressivos e ansiosos durante a gravidez, história de depressão, eventos de vida estressantes durante a gravidez, complicações médicas no pós-parto e ausência de suporte social ${ }^{18,19}$.

O diagnóstico precoce da DPP e uma intervenção terapêutica adequada poderiam diminuir os prejuízos sobre a mãe e os efeitos negativos de longo prazo da DPP sobre a criança e a família ${ }^{8}$. Infelizmente, apesar da gravidade das manifestações, somente uma minoria das pacientes acometidas por DPP recebe tratamento adequado ${ }^{12}$.

No presente estudo, objetivamos comparar mulheres com e sem DPP dentre puérperas selecionadas aleatoriamente a partir dos partos ocorridos em uma maternidade de Belo Horizonte (MG). Utilizamos uma entrevista diagnóstica estruturada para o diagnóstico e uma entrevista semiestruturada para investigar características sociodemográficas, clínicas e psicossociais que estudos prévios mostraram como potencialmente associadas à DPP. Os resultados coletados foram comparados aos dados descritos na literatura.

\section{Material e métodos}

Foram incluídas neste estudo 245 mulheres selecionadas aleatoriamente no período de agosto de 2005 a dezembro de 2006 na Maternidade Santa Fé, uma instituição de saúde privada de Belo Horizonte (MG). Uma descrição mais detalhada da amostra e do processo de seleção pode ser encontrada em estudo prévio dos autores $^{12}$. A inclusão se deu após uma explicação sobre o estudo e a assinatura de um termo de consentimento livre e esclarecido. O projeto foi aprovado pela Comitê de Ética em Pesquisa da Universidade Federal de Minas Gerais (processo n ${ }^{\circ} 227 / 05$ ).

Cada participante recebeu uma visita domiciliar entre 40 e 90 dias após o parto (média de 58,2 $\pm 9,5$ dias), na qual foi realizada uma entrevista semiestruturada, elaborada pelos autores, para obter dados psicossociais, clínicos e sociodemográficos sobre a mãe e a criança. Os seguintes dados foram investigados: idade, dias transcorridos após o parto, peso e altura(s) da(s) criança(s) no nascimento e após 1 mês (avaliados pelo boletim de parto e dados da consulta com o pediatra no primeiro mês de vida), situação conjugal, número de gestações e de partos, atividade laboral, histórico de depressão, quadro depressivo em pós-parto anterior, história familiar de doença psiquiátrica, ocorrência de complicações obstétricas, de evento estressante e de sintomas depressivos e/ou ansiosos durante a gravidez, complicações no pós-parto em relação à paciente ou à criança, suporte social no pós-parto (ajuda nos cuidados da criança), dificuldade financeira, estresse no cuidado da criança, passado de sintomas depressivos e/ou ansiosos no período pré-menstrual. Nessa visita, aplicamos também uma entrevista diagnóstica estruturada, a Mini Neuropsychiatric Interview (MINI-Plus) ${ }^{20}$, para a identificação de pacientes com diagnóstico de depressão maior.

O teste do qui-quadrado foi usado para comparar a frequência das variáveis categoriais, e o teste $t$ de Student foi usado para comparar variáveis contínuas. Os resultados foram considerados significativos quando $\mathrm{p} \leq 0.05$.

\section{Resultados}

Das 245 puérperas, 66 mulheres (26,9\%) apresentaram diagnóstico de depressão maior segundo critérios do Diagnostic and Statistical Manual of Mental Disorders, 4th edition (DSM-IV), sendo incluídas no grupo de portadoras de DPP; as demais 179 mulheres $(73,1 \%)$ não tiveram o diagnóstico de depressão estabelecido pelo MINI-Plus, sendo, portanto, incluídas no grupo de não portadoras de DPP.

Na Tabela 1, é possível observar que os dados sociodemográficos foram estatisticamente semelhantes entre as mulheres que receberam diagnóstico de DPP e aquelas que não receberam esse diagnóstico.

Também não observamos diferenças estatisticamente significativas no peso e altura das crianças, tanto ao nascimento quanto após 1 mês de vida, entre os grupos de mulheres com e sem DPP. 
Tabela 1 - Dados sociodemográficos das puérperas com e sem depressão pós-parto

\begin{tabular}{|c|c|c|c|}
\hline Dados sociodemográficos & $\mathrm{DPP}+(n=66)$ & DPP- & $\mathbf{p}$ \\
\hline Momento da avaliação (dias após o parto) & $58,26 \pm 10,51$ & $58,23 \pm 9,80$ & $0,98^{*}$ \\
\hline Idade $($ anos $) \pm$ DP & $30,24 \pm 5,60$ & $30,92 \pm 5,90$ & $0,42^{\dagger}$ \\
\hline Número de gestações & $1,62 \pm 0,97$ & $1,7 \pm 0,88$ & $0,53^{\ddagger}$ \\
\hline \multicolumn{4}{|l|}{ Escolaridade } \\
\hline Ensino médio & 36 & 79 & \\
\hline Ensino superior & 30 & 100 & $0,14^{\S}$ \\
\hline \multicolumn{4}{|l|}{ Estado civil } \\
\hline Casada & 53 & 142 & \\
\hline Não casada & 13 & 37 & $0,86^{\|}$ \\
\hline \multicolumn{4}{|l|}{ Situação laborativa } \\
\hline Trabalha & 25 & 56 & \\
\hline Não trabalha & 41 & 123 & $0,33^{\pi}$ \\
\hline
\end{tabular}

${ }^{*}$ Teste $\mathrm{t}=0,21 ;{ }^{\dagger}$ teste $\mathrm{t}=0,8 ;{ }^{\ddagger}$ teste $\mathrm{t}=0,64 ;{ }^{\circledR} \chi^{2}=6,72 ;{ }^{\prime \prime} \chi^{2}=0,75 ;{ }^{\circledR} \chi^{2}=0,96$.

Dentre as características clínicas, história familiar de doença psiquiátrica $\left(\chi^{2}=4,11, \mathrm{p}=0,13\right)$ e histórico de $\operatorname{DPP}\left(\chi^{2}=\right.$ $3,76, p=0,08)$ não mostraram diferença estatisticamente significativa entre as mulheres com e sem diagnóstico de DPP.
A Tabela 2 mostra os dados para os quais foram observadas diferenças estatisticamente significativas entre as mulheres com e sem diagnóstico de DPP.

Tabela 2 - Dados clínicos com diferença significativa entre as mulheres com e sem diagnóstico de depressão pós-parto

\begin{tabular}{|c|c|c|c|}
\hline Dados clínicos & $\mathrm{DPP}+(n=66)$ & DPP- & $\mathbf{p}$ \\
\hline Escore total na Escala de Depressão Pós-Natal de Edimburgo & $15,35 \pm 5,35$ & $5,90 \pm 3,28$ & $0,0001^{*}$ \\
\hline \multicolumn{4}{|l|}{ Complicações obstétricas na gravidez } \\
\hline Sim & 27 & 47 & \\
\hline Não & 39 & 132 & $0,03^{\dagger}$ \\
\hline \multicolumn{4}{|l|}{ Estresse na gravidez } \\
\hline Não & 13 & 70 & \\
\hline Um estressor & 26 & 70 & \\
\hline Mais de um estressor & 27 & 39 & 0,004 \\
\hline \multicolumn{4}{|l|}{ Sintomas depressivos na gravidez } \\
\hline Sim & 53 & 88 & \\
\hline Não & 13 & 91 & $0,0001^{\S}$ \\
\hline \multicolumn{4}{|l|}{ Sintomas ansiosos na gravidez } \\
\hline Sim & 53 & 97 & \\
\hline Não & 13 & 82 & $0,0001^{\|}$ \\
\hline \multicolumn{4}{|l|}{ Complicações no pós-parto } \\
\hline Sim & 40 & 134 & \\
\hline Não & 26 & 45 & $0,01^{\uparrow}$ \\
\hline \multicolumn{4}{|l|}{ Suporte social no pós-parto } \\
\hline Sim & 47 & 162 & \\
\hline Não & 19 & 17 & $0,0001^{\text {** }}$ \\
\hline \multicolumn{4}{|l|}{ Dificuldade financeira } \\
\hline Sim & 23 & 27 & \\
\hline Não & 43 & 152 & $0,001^{\dagger}$ \\
\hline \multicolumn{4}{|l|}{ Sintomas depressivos ou ansiosos no período pré-menstrual } \\
\hline Sim 1 & 48 & 93 & \\
\hline Não & 18 & 86 & 0,004 \\
\hline \multicolumn{4}{|l|}{ Sintomas depressivos no pós-parto } \\
\hline Sim & 60 & 110 & \\
\hline Não & 6 & 69 & $0,0001^{\S \S}$ \\
\hline \multicolumn{4}{|l|}{ Quadro depressivo prévio } \\
\hline Sim & 31 & 40 & \\
\hline Não & 35 & 139 & $0,0001^{|\||}$ \\
\hline \multicolumn{4}{|l|}{ Estresse no cuidado da criança } \\
\hline Sim & 43 & 77 & \\
\hline Não & 23 & 102 & $0,0001^{\pi}$ \\
\hline
\end{tabular}




\section{Discussão}

Em nosso estudo, observamos similaridade nos dados demográficos e de peso e altura dos recém-nascidos de mulheres com e sem DPP, porém encontramos diferenças significativas em várias variáveis clínicas e psicossociais.

Assim como em outros estudos ${ }^{18}$, não encontramos associação estatisticamente significativa entre as variáveis sociodemográficas (média de idade, estado civil, nível educacional, número de gestações e de partos e trabalho ou não fora de casa) e a presença ou ausência de DPP.

Apesar de alguns estudos mostrarem um aumento no risco de DPP entre mulheres solteiras ${ }^{21}$, outros, como este, não confirmam essa relação ${ }^{15,18,22,23}$. Da mesma forma, alguns estudos mostraram associações entre primeira gravidez e DPP ${ }^{21}$, o que não foi evidenciado neste estudo e em ou$\operatorname{tros}^{15,24}$. Também não observamos diferenças em relação à situação laborativa das mães. Conforme descrito na literatura, observamos que a presença de dificuldades financeiras têm uma relação mais direta com o risco de desenvolver DPP do que trabalhar ou não fora de casa ${ }^{15}$.

Inúmeros outros fatores foram, entretanto, associados ao diagnóstico de DPP em nossa amostra: história de quadro depressivo, presença de complicações obstétricas durante a gravidez, vivência de estresse na gravidez, presença de sintomas depressivos e/ou ansiosos durante a gravidez, ocorrência de complicações no pós-parto, ausência de suporte social no pós-parto, dificuldades financeiras e/ou estresse no cuidado da criança no pós-parto, história de sintomas depressivos ou ansiosos no período pré-menstrual e presença de sintomas depressivos no pós-parto.

Alguns autores afirmam que o principal fator de risco para a DPP é a susceptibilidade individual à doença $\mathrm{a}^{25}$, o que pode ser inferido pela história de quadro depressivo em nossa amostra. Neste estudo, replicamos os dados da literatura, pois mostramos que significativamente mais mulheres com DPP tinham história de depressão quando comparadas com mulheres sem DPP. A presença de sintomas depressivos durante a gravidez também tem sido considerada um fator de risco para a $\mathrm{DPP}^{21}$, o que confirmamos neste estudo ao demonstrar diferença estatisticamente significativa para essa variável entre as mulheres com e sem DPP. Também pudemos observar maior frequência na presença de sintomas ansiosos durante a gravidez nas mulheres com DPP, conforme descrito na literatura ${ }^{26}$.

Com relação ao impacto de complicações obstétricas na DPP, alguns estudos mostraram risco aumentado para a doença em mulheres que tiveram mais complicações obstétricas $^{21,27}$. Neste estudo, de fato, houve diferença significativa na presença de complicações obstétricas durante a gravidez entre mulheres com e sem DPP.

A associação entre eventos estressantes e transtornos do humor descrita na literatura ${ }^{28}$ também foi testada. Encontramos, no grupo de pacientes com DPP, o relato da presença de evento estressor em pelo menos uma das esferas pesquisa- das em $39,4 \%$ das mulheres, e em dois ou mais aspectos em $40,9 \%$. No grupo de pacientes sem DPP, 39, $1 \%$ das mulheres relataram ausência de evento estressor na gravidez, e 39,1\% relataram presença de evento estressor em pelo menos um dos aspectos pesquisados. As diferenças foram estatisticamente significativas, confirmando a associação entre eventos de vida estressante na gravidez e o aparecimento de DPP. Alguns autores hipotetizam se o parto seria um estressor por si só, e se mulheres vulneráveis à depressão se tornariam deprimidas nesse período, principalmente quando esse estresse é potencializado pela presença de outro evento de vida estressante ${ }^{29}$.

A presença de complicações no pós-parto, seja em relação à saúde da mãe, seja em relação à saúde da criança, também aumenta o nível de estresse nesse período ${ }^{29}$. Nosso estudo confirmou essa associação, sendo que 39,4\% das mulheres com DPP e 25,1\% daquelas sem DPP relataram presença de complicações no pós-parto.

A vivência de estresse em relação aos cuidados da criança também foi relacionada ao aumento do risco de $\mathrm{DPP}^{23}$. Observamos diferença significativa entre as mulheres com e sem DPP em relação à percepção de pelo menos um tipo de estresse nos cuidados da criança. A questão que se coloca é de se a própria percepção subjetiva da paciente no que diz respeito à presença de dificuldades em relação a algum aspecto do cuidado da criança não seria já uma consequência do seu estado depressivo, em vez de um fator causal.

Outra questão estudada foi o suporte social no pós-parto. No grupo de pacientes com DPP, $28,8 \%$ das mulheres negaram presença de suporte social no pós-parto; já no grupo sem DPP, apenas 9,5\% das pacientes fizeram esse relato. A diferença entre os grupos foi estatisticamente significativa, confirmando os dados descritos na literatura, de que a ajuda e o apoio emocional nos cuidados pós-natais são fatores protetores em relação à $\mathrm{DPP}^{25}$. A adaptação ao papel de mãe e à demanda da criança exige habilidades que seriam facilitadas pela presença de ajuda nos cuidados, diminuindo sentimentos de medo e insegurança, e também de culpa e incapacidade.

Alguns estudos tentaram esclarecer a relação entre história de síndrome pré-menstrual e ocorrência de DPP ${ }^{19,24}$. Neste estudo, não utilizamos escala específica para a pesquisa de síndrome pré-menstrual, porém foi questionada a presença de sintomas depressivos ou ansiosos no período pré-menstrual. No grupo de pacientes com DPP, 72,7\% das mulheres reportaram história de sintomas depressivos ou ansiosos no período pré-menstrual, comparado com 51,9\% no grupo de pacientes sem DPP, sendo a diferença estatisticamente significativa.

Da mesma forma, não usamos escala específica para a pesquisa de disforia do pós-parto, porém houve questionamento sobre a presença de sintomas depressivos na primeira semana pós-parto que tenham desaparecido em até 10 dias. Apesar de não ser possível caracterizar tais sintomas como um quadro nosológico, encontramos uma alta prevalência de retornos positivos para esse questionamento, principalmente 
no grupo de mulheres que apresentaram DPP, sugerindo uma relação entre esses sintomas no pós-parto imediato e o aparecimento da doença.

Em relação à história familiar de doença psiquiátrica, não houve diferença estatisticamente significativa entre o grupo de pacientes com e sem DPP. Tendo em vista que não foi aplicado um questionário apropriado para o estudo desse fator, há uma limitação na interpretação desse resultado. Estudo prévio realizado por O'Hara et al. ${ }^{29}$, porém, também não encontrou associação entre história familiar de depressão e DPP.

História de DPP foi confirmada $12,1 \%$ das mulheres no grupo de pacientes com DPP e em 5\% das mulheres no grupo sem DPP. Essa diferença não foi estatisticamente significativa, o que corrobora o estudo feito por Cooper $\&$ Murray $^{30}$, que sugeriu que a presença de DPP por si só não é considerada um fator de risco para outros episódios de DPP futuros.

\section{Conclusão}

A alta prevalência de DPP e suas possíveis consequências para a paciente e para a criança reforçam a importância de seu estudo. A etiopatologia da doença não foi ainda bem definida e provavelmente envolve fatores biopsicossociais. Assim, o estabelecimento de possíveis fatores de risco contribui para uma melhor compreensão da doença e para a elaboração de estratégias de prevenção e diagnóstico precoce.

Em nosso estudo, as seguintes variáveis foram identificadas como possíveis fatores de risco para DPP: história de quadro depressivo, presença de complicações obstétricas durante a gravidez, vivência de situação de estresse na gravidez, presença de sintomas depressivos ou ansiosos durante a gravidez, ocorrência de complicações no pós-parto, ausência de suporte social no pós-parto, presença de dificuldades financeiras no pós-parto, presença de estresse no cuidado da criança no pós-parto, história de sintomas depressivos e ansiosos no período pré-menstrual, presença de sintomas depressivos no pós-parto. Esses achados sugerem que a etiopatogenia da doença envolve diversos fatores, entre eles uma vulnerabilidade individual a quadros depressivos e uma maior vulnerabilidade ao estresse relacionado à gravidez $\mathrm{e}$ ao pós-parto.

\section{Referências}

1. Murray L, Cooper PJ. Postpartum depression and child development. Psychol Med. 1997;27(2):253-60.

2. Nielsen FD, Videbech P, Hedegaard M, Dalby SJ, Secher NJ. Postpartum depression: identification of women at risk. BJOG. 2000;107(10):1210-7.

3. Murray L, Cooper PJ, Wilson A, Romaniuk, H. Controlled trial of the short- and long-term effect of psychological treatment of post-partum depression. 2. Impact on the mother-child relationship and child outcome. Br J Psychiatry. 2003;182:420-7.
4. Kumar RC. "Anybody's child": severe disorders of mother-to-infant bonding. Br J Psychiatry. 1997;171:175-81.

5. Kurstjens S, Wolke D. Effects of maternal depression on cognitive development of children over the first 7 years of life. J Child Psychol Psychiatry. 2001;42:623-36.

6. Rahman A, Iqbal Z, Bunn J, Lovel H, Harrington R. Impact of maternal depression on infant nutritional status and illness. Arch Gen Psychiatry. 2004;61(9):946-52.

7. Teti DM, Gelfand DM, Messinger DS, Isabella R. Maternal depression and the quality of early attachment: an examination of infants, preschoolers, and their mothers. Dev Psychol. 1995;31(3):364-76.

8. Montgomery SA, Asberg M. A new depression scale designed to be sensitive to change. Br J Psychiatry. 1979;134:382-9.

9. Silva VA, Santos ARM, Carvalho MS, Martins MLP, Teixeira NA. Prenatal and postnatal depression among low-income Brazilian women. Braz J Med Biol Res. 1998;31(6):799-804.

10. Santos MFS, Martins FC, Pasquali L. Escalas de auto-avaliação de depressão pós-parto: um estudo no Brasil. Rev Psiquiatr Clin. 1999;26:32-40.

11. Tannous L, Gigante LP, Fuchs SC, Busnello ED. Postnatal depression in southern Brazil: prevalence and its demographic and socioeconomic determinants. BMC Psychiatry. 2008;8:1. doi: 10.1186/1471-244X-8-1.

12. Figueira P, Correa H, Malloy-Diniz L, Romano-Silva MA. Edinburgh Postnatal Depression Scale for screening in the public health system. Rev Saude Publica. 2009;43(suppl 1):79-84

13. Cantilino A, Zambaldi CF, Albuquerque T, Paes JA, Montenegro AC, Sougey EB. Postpartum depression in Recife - Brazil: prevalence and association with bio-socio-demographic factors. J Bras Psiquiatr. 2010;59(1):1-9.

14. Halbreich U. Postpartum disorders: multiple interacting underlying mechanisms and risk factors. J Affect Disord. 2005;88(1):1-7.

15. O'Hara MW, Swain AM. Rates and risk of postpartum depression--a meta-analysis. Int Rev Psychiatry. 1996;8(1):37-54.

16. Bloch M, Schmidt PJ, Danaceau M, Murphy J, Nieman L, Rubinow DR. Effects of gonadal steroids in women with a history of postpartum depression. Am J Psychiatry. 2000;157(6):924-30.

17. Figueira P, Malloy-Diniz L, Campos S, Miranda DM, Romano-Silva MA, Neves FS, et al. An association study between the Val66Met polymorphism 5 of the BDNF gene and postpartum depression. Arch Womens Ment Health. 2010;13(3):285-9.

18. Boyce MP. Risk factors for postnatal depression: a review and risk factors in Australian populations. Arch Womens Ment Health. 2003;6(Suppl 2):43-50.

19. Bloch M, Rotenberg N, Koren D, Klein E. Risk factors associated with the development of postpartum mood disorders. J Affect Disord. 2005;88(1):9-18.

20. Amorim P. The Mini Neuropsychiatric Interview (MINI): desenvolvimento de um questionário diagnóstico padronizado breve para avaliação dos transtornos mentais do DSM-IV e da CID-10. Rev Bras Psiquiatr. 2003;22(3):26-39.

21. Vesga-López O, Blanco C, Keyes K, Olfson M, Grant BF, Hasin DS. Psychiatric disorders in pregnant and postpartum women in the United States. Arch Gen Psychiatry. 2008;65(7):805-15.

22. Halbreich U, Karkun S. Cross-cultural and social diversity of prevalence of postpartum depression and depressive symptoms. J Affect Disord. 2006;91(2):97-111.

23. Areias M, Kumar R, Barros H, Figueiredo E. Comparative incidence of depression in women and men, during pregnancy and after childbirth: validation of the Edinburgh Postnatal Depression Scale in Portuguese mothers. Br J Psychiatry. 1996;169:30-5.

24. Posner NA, Unterman RR, Williams KN, Williams GH. Screening for postpartum depression. An antepartum questionnaire. J Reprod Med. 1997;42(4):207-15.

25. Riecher-Rössler A, Fallahpour MH. Postpartum depression: do we still need this diagnostic term? Acta Psychiatr Scand Suppl. 2003;418:51-6.

26. Glover V, O'Connor TG. Effects of antenatal stress and anxiety: implications for development and psychiatry. Br J Psychiatry. 2002;180:389-91.

27. Fisher J, Astbury J, Smith A. Adverse psychological impact of operative obstetric interventions: a prospective longitudinal study. Aust N Z J Psychiatry. 1997;31(5):728-38.

28. Kendler KS, Thornton LM, Gardner CO. Stressful life events and previous episodes in the etiology of major depression in women: an evaluation of the "kindling" hypothesis. Am J Psychiatry. 2000;157(8):1243-51.

29. O'Hara MW, Schlechte JA, Lewis DA, Varner MW. Controlled prospective study of postpartum mood disorders: psychological, environmental, and hormonal variables. J Abnorm Psychol. 1991;100(1):63-73.

30. Cooper PJ, Murray L. The course and recurrence of postnatal depression. Br J Psychiatry. 1995;166:191-5. 